\title{
Boundedness in Lebesgue spaces of Riesz potentials on commutative hypergroups
}

\author{
Mubariz G. Hajibayov \\ National Aviation Academy, Baku, Azerbaijan \\ and \\ Institute of Mathematics and Mechanics, Baku, Azerbaijan \\ Email: hajibayovm@yahoo.com
}

Copyright (c) 2015 M. G. Hajibayov. This is an open access article distributed under the Creative Commons Attribution License, which permits unrestricted use, distribution, and reproduction in any medium, provided the original work is properly cited.

\begin{abstract}
In the present paper we consider Riesz potentials on commutative hypergroups and prove the boundedness of these potentials from $L^{p}(K, \lambda)$ to $L^{q}(K, \lambda)$. We also prove the inequality from $L^{1}(K, \lambda)$ to weak $L^{q}(K, \lambda)$ for Riesz potentials on commutative hypergroups.
\end{abstract}

Keywords: Hardy-Littlevood maximal function, hypergroup, Riesz potential.

\section{Introduction}

For $0<\alpha<n$, the operator

$$
R_{\alpha} f(x)=\int_{R^{n}}|x-y|^{\alpha} f(y) d y
$$

is called a classical Riesz potential.

By the classical Hardy-Littlewood-Sobolev theorem, if $1<p<\infty$ and $\alpha p<n$, then $R_{\alpha} f$ is an operator of strong type $(p, q)$, where $\frac{1}{q}=\frac{1}{p}-\frac{\alpha}{n}$. If $p=1$, then $R_{\alpha} f$ is an operator of weak type $(1, q)$, where $\frac{1}{q}=1-\frac{\alpha}{n}$ (see [16], [24]).

The Hardy-Littlewood-Sobolev theorem is an important result in fractional integral theory and potential theory. There are a lot of generalizations and analogues of this theorem. The Hardy-Littlewood-Sobolev theorem was proved for Riesz potentials associated to doubling measures in [18] and nondoubling measures in [19], [5]. In [3] and [11], generalized potential-type integral operators were considered and $(p, q)$ properties of these operators were proved. In [21], [22], [13], [12] the Hardy-Littlewood-Sobolev theorem was extended to Orlicz and Musielak-Orlicz spaces for generalized Riesz potentials. The analogues of Hardy-Littlewood-Sobolev theorem on the different hypergroups were considered in [8], [9], [10], [4], [14]. The strong type $(p, q)$ inequality for Riesz potentials on commutative hypergroups was given in [15] with the condition $\lambda B(e, r)=C r^{N}$.

In this paper, we prove the boundedness of Riesz potentials on commutative hypergroups from $L^{p}(K, \lambda)$ to $L^{q}(K, \lambda)$ in the case of upper Ahlfors $N$-regular measure. We also prove the inequality from $L^{1}(K, \lambda)$ to weak $L^{q}(K, \lambda)$ for Riesz potentials on commutative hypergroups. 


\section{Preliminaries}

Let $K$ be a set. A function $\rho: K \times K \rightarrow[0, \infty)$ is called quasi-metric if:

1. $\rho(x, y)=0 \Leftrightarrow x=y$;

2. $\rho(x, y)=\rho(y, x)$;

3. there exists a constant $c \geq 1$ such that for every $x, y, z \in K$

$$
\rho(x, y) \leq c(\rho(x, z)+\rho(z, y)) .
$$

Let all balls $B(x, r)=\{y \in K: \rho(x, y)<r\}$ be $\lambda$-measurable and assume that the measure $\lambda$ fulfils the doubling condition

$0<\lambda B(x, 2 r) \leq D \lambda B(x, r)<\infty$.

A space $(K, \rho, \lambda)$ which satisfies all conditions mentioned above is called a space of homogeneous type (see [2]).

In the theory of locally compact groups there arise certain spaces which, though not groups, have some of the structure of groups. Often, the structure can be expressed in terms of an abstract convolution of measures on the space.

A hypergroup $(K, *)$ consists of a locally compact Hausdorff space $\mathrm{K}$ together with a bilinear, associative, weakly continuous convolution on the Banach space of all bounded regular Borel measures on $K$ with the following properties:

1. For all $x, y \in K$, the convolution of the point measures $\delta_{x} * \delta_{y}$ is a probability measure with compact support.

2. The mapping: $K \times K \rightarrow \mathcal{C}(K),(x, y) \mapsto \operatorname{supp}\left(\delta_{x} * \delta_{y}\right)$ is continuous with respect to the Michael topology on the space $\mathcal{C}(K)$ of all nonvoid compact subsets of $K$, where this topology is generated by the sets

$$
U_{V, W}=\{L \in \mathcal{C}(K): L \cap V \neq \emptyset, L \subset W\}
$$

with $V, W$ open in $K$.

3. There is an identity $e \in K$ with $\delta_{e} * \delta_{x}=\delta_{x} * \delta_{e}=\delta_{x}$ for all $x \in K$.

4. There is a continuous involution $*$ on $K$ such that

$$
\left(\delta_{x} * \delta_{y}\right)^{*}=\delta_{y^{*}} * \delta_{x^{*}}
$$

and $e \in \operatorname{supp}\left(\delta_{x} * \delta_{y}\right) \Leftrightarrow x=y^{*}$ for $x, y \in K$ (see [17], [23], [1], [20], [7] ).

A hypergroup $K$ is called commutative if $\delta_{x} * \delta_{y}=\delta_{y} * \delta_{x}$ for all $x, y \in K$. It is well known that every commutative hypergroup $K$ possesses a Haar measure which will be denoted by $\lambda$ (see [23]). That is, for every Borel measurable function $f$ on $K$,

$$
\int_{K} f\left(\delta_{x} * \delta_{y}\right) d \lambda(y)=\int_{K} f(y) d \lambda(y) \quad(x \in K) .
$$

Define the generalized translation operators $T^{x}, x \in K$, by

$$
T^{x} f(y)=\int_{K} f d\left(\delta_{x} * \delta_{y}\right)
$$

for all $y \in K$. If $K$ is a commutative hypergroup, then $T^{x} f(y)=T^{y} f(x)$ and the convolution of two functions is defined by

$$
(f * g)(x)=\int_{K} T^{x} f(y) g\left(y^{*}\right) d \lambda(y) .
$$

Let $(K, *)$ be a commutative hypergroup, with quasi-metric $\rho$, Haar measure $\lambda$ and $N \in(0, \infty)$. We will say $K$ is called upper Ahlfors $N$-regular by an identity, if there exists a constant $C>0$, not depending $r>0$, such that

$\lambda B(e, r) \leq C r^{N}$ 
Let $p>0$. $\operatorname{By} L^{p}(K, \lambda)$ denote a class of all $\lambda$-measurable functions $f: K \rightarrow(-\infty,+\infty)$ with $\|f\|_{L^{p}(K, \lambda)}=$ $\left(\int_{K}|f(x)|^{p} d \lambda(x)\right)^{\frac{1}{p}}<\infty$.

Let $T$ be a linear operator from $L^{p}(K, \lambda)$ to $L^{q}(K, \lambda)$, where $p, q \in(0, \infty)$. $T$ is said to be an operator of strong type $(p, q)$ on $(K, \lambda)$, if there exists a positive constant $C$ such that

$$
\|T f\|_{L^{p}(K, \lambda)} \leq C_{p}\|f\|_{L^{p}(K, \lambda)},
$$

If for arbitrary $\beta>0$ and $f \in L^{p}(K, \lambda)$

$$
\lambda\{x: T f(x)>\beta\} \leq\left(\frac{C}{\beta}\|f\|_{L^{1}(K, \lambda)}\right)^{q}, \beta>0
$$

then $T$ is called an operator of weak type $(p, q)$ on $(K, \lambda)$.

The notation $\chi_{A}(x)$ denotes the characteristic function of set $A$.

Let $(K, *)$ be a commutative hypergroup, with quasi-metric $\rho$, Haar measure $\lambda$, upper Ahlfors $N$-regular by an identity. Define Hardy-Littlewood maximal function

$M f(x)=\sup _{r>0} \frac{1}{\lambda B(e, r)}\left(|f| * \chi_{B(e, r)}\right)(x)$

and Riesz potential

$I_{\alpha} f(x)=\left(\rho(e, \cdot)^{\alpha-N} * f\right)(x), 0<\alpha<N$

on commutative hypergroup $(K, *)$ equipped with the quasi-metric $\rho$.

\section{Main results}

In this section we formulate the main results of this paper.

Theorem 3.1 Let $(K, *)$ be a commutative hypergroup, with quasi-metric $\rho$ and doubling Haar measure $\lambda$, upper Ahlfors $N$-regular by an identity and let $0<\alpha<N, 1 \leq p<\frac{N}{\alpha}$ If $f \in L^{p}(K)$, then the integral

$$
I_{\alpha} f(x)=\int_{X} T^{x} \rho(e, y)^{\alpha-N} f\left(y^{*}\right) d \lambda(y)
$$

is absolutely convergent for almost every $x \in K$.

Proof Let $f \in L^{p}(K, \lambda)$ and $1 \leq p<\frac{N}{\alpha}$. Write $I_{\alpha} f(x)$ in the form

$$
\begin{gathered}
I_{\alpha} f(x)=\int_{B(e, 1)} \rho(e, y)^{\alpha-N} T^{x} f\left(y^{*}\right) d \lambda(y) \\
+\int_{K \backslash B(e, 1)} \rho(e, y)^{\alpha-N} T^{x} f\left(y^{*}\right) d \lambda(y)=J_{1}(x)+J_{2}(x) .
\end{gathered}
$$

Let us estimate $J_{1}(x)$. It is clear that

$$
\left|J_{1}(x)\right| \leq \int_{K} \rho(e, y)^{\alpha-N} \chi_{B(e, 1)}(y) T^{x}\left|f\left(y^{*}\right)\right| d \lambda(y)
$$

By Young's inequality

$$
\begin{gathered}
\left\|J_{1}(\cdot)\right\|_{L^{p}(K, \lambda)} \leq\left\|\rho(e, \cdot)^{\alpha-N} \chi_{B(e, 1)}(\cdot)\right\|_{L^{1}(K, \lambda)}\left\|T^{x} f\right\|_{L^{p}(K, \lambda)} \\
\leq C\left\|\rho(e, \cdot)^{\alpha-N} \chi_{B(e, 1)}(\cdot)\right\|_{L^{1}(K, \lambda)}\|f\|_{L^{p}(K, \lambda)}
\end{gathered}
$$


and

$$
\begin{gathered}
\left\|\rho(e, \cdot)^{\alpha-N} \chi_{B(e, 1)}(\cdot)\right\|_{L^{1}(K, \lambda)}=\int_{B(e, 1)} \rho(e, y)^{\alpha-N} d \lambda(y) \\
\leq \sum_{k=1_{2^{-k} \leq \rho(e, y)<2^{-k+1}}}^{\infty} \rho(e, y)^{\alpha-N} d \lambda(y) \\
\leq \sum_{k=1}^{\infty}\left(2^{-k}\right)^{\alpha-N} \int_{\rho(e, y)<2^{-k+1}} d \lambda(y) \\
\leq C \sum_{k=1}^{\infty} 2^{(N-\alpha) k} 2^{N(-k+1)}<C
\end{gathered}
$$

Then

$$
\left\|J_{1}(\cdot)\right\|_{L^{p}(K, \lambda)} \leq C\|f\|_{L^{p}(K, \lambda)}
$$

e.g. $J_{1}(x)$ is absolutely convergent almost every $x \in K$.

By Hölder's inequality we have

$$
\begin{gathered}
\left|J_{2}(x)\right| \leq \int_{K \backslash B(e, 1)} \rho(e, y)^{\alpha-N} T^{x}\left|f\left(y^{*}\right)\right| d \lambda(y) \\
\leq\left\|T^{x} f(\cdot)\right\|_{L^{p}(K, \lambda)}\left(\int_{K \backslash B(e, 1)} \rho(e, y)^{(\alpha-N) p^{\prime}} d \lambda(y)\right)^{\frac{1}{p^{\prime}}} \\
\leq C\|f\|_{L^{p}(K, \lambda)}\left(\int_{K \backslash B(e, 1)} \rho(e, y)^{(\alpha-N) p^{\prime}} d \lambda(y)\right)^{\frac{1}{p^{\prime}}}
\end{gathered}
$$

and

$$
\begin{gathered}
\int_{K \backslash B(e, 1)} \rho(e, y)^{(\alpha-N) p^{\prime}} d \lambda(y) \\
\leq \sum_{k=0}^{\infty} \int_{2^{k}<\rho(e, y) \leq 2^{k+1}} \rho(e, y)^{(\alpha-N) p^{\prime}} d \lambda(y) \\
\leq \sum_{k=0}^{\infty} 2^{(N-\alpha) p^{\prime} k} \int_{\rho(e, y) \leq 2^{k+1}} d \lambda(y) \\
\leq \sum_{k=0}^{\infty} 2^{(N-\alpha) p^{\prime} k} 2^{(k+1) N}<C
\end{gathered}
$$

Hence for $1 \leq p<\frac{N}{\alpha}$

$$
\left|J_{2}(x)\right| \leq C\|f\|_{L^{p}(K, \lambda)}
$$

Thus for all functions $f \in L^{p}(K, \lambda), 1 \leq p<\frac{N}{\alpha}$ the fractional integrals $I_{\alpha} f(x)$ are absolutely convergent for almost every $x \in K$. The theorem is proved.

Theorem 3.2 Let $(K, *)$ be a commutative hypergroup, with quasi-metric $\rho$ and doubling Haar measure $\lambda$, upper Ahlfors $N$-regular by an identity. Assume $0<\alpha<N, 1<p<\frac{N}{\alpha}, \frac{1}{p}-\frac{1}{q}=\frac{\alpha}{N}$ and Hardy-Littlewood maximal function (3) is an operator of strong type $(p, p)$ on $(K, *)$. Then the Riesz potential (4) is an operator of strong type $(p, q)$ on $(K, *)$. 
Proof Split $I_{\alpha} f(x)$ in the standard way

$$
\begin{gathered}
I_{\alpha} f(x)=\int_{B(e, r)} \rho(e, y)^{\alpha-N} T^{x} f\left(y^{*}\right) d \lambda(y)+\int_{K \backslash B(e, r)} \rho(e, y)^{\alpha-N} T^{x} f\left(y^{*}\right) d \lambda(y) \\
=U_{1}(x, r)+U_{2}(x, r) .
\end{gathered}
$$

Then for $U_{1}(x, r)$ we have the estimate

$$
\begin{gathered}
\left|U_{1}(x, r)\right| \leq \int_{B(e, r)} \rho(e, y)^{\alpha-N} T^{x}\left|f\left(y^{*}\right)\right| d \lambda(y) . \\
\leq \sum_{k=1}^{\infty} \int_{2^{-k} r \leq \rho(e, y)<2^{-k+1} r} \rho(e, y)^{\alpha-N} T^{x}\left|f\left(y^{*}\right)\right| d \lambda(y) \\
\leq \sum_{k=1}^{\infty}\left(2^{-k} r\right)^{\alpha-N} \int_{\rho(e, y)<2^{-k+1} r} T^{x}\left|f\left(y^{*}\right)\right| d \lambda(y) \\
=\sum_{k=1}^{\infty}\left(2^{-k} r\right)^{\alpha-N} \int_{B\left(e, 2^{-k+1} r\right)} T^{x}\left|f\left(y^{*}\right)\right| d \lambda(y) \\
\leq C r^{\alpha} M f(x) .
\end{gathered}
$$

Therefore it follows that

$\left|U_{1}(x, r)\right| \leq C r^{\alpha} M f(x)$,

where $C>0$ does not depend $f, x$ and $r$.

Estimate $U_{2}(x, r)$. By Hölder's inequality we have

$$
\left|U_{2}(x, r)\right| \leq\left(\int_{K \backslash B(e, r)}\left|T^{x} f\left(y^{*}\right)\right|^{p} d \lambda(y)\right)^{\frac{1}{p}}\left(\int_{K \backslash B(e, r)} \rho(e, y)^{(\alpha-N) p^{\prime}} d \lambda(y)\right)^{\frac{1}{p^{\prime}}} .
$$

Here

$$
\begin{gathered}
\left(\int_{K \backslash B(e, r)} \rho(e, y)^{(\alpha-N) p^{\prime}} d \lambda(y)\right)^{\frac{1}{p^{\prime}}} \\
=\left(\sum_{k=0}^{\infty} \int_{2^{k} r \leq \rho(e, y)<2^{k+1} r} \rho(e, y)^{(\alpha-N) p^{\prime}} d \lambda(y)\right)^{\frac{1}{p^{\prime}}} \\
\leq\left(\sum_{k=0}^{\infty}\left(2^{k} r\right)^{(\alpha-N) p^{\prime}} \int_{\rho(e, y)<2^{k+1} r} d \lambda(y)\right)^{\frac{1}{p^{\prime}}} \\
\leq C\left(\sum_{k=0}^{\infty}\left(2^{k} r\right)^{(\alpha-N) p^{\prime}}\left(2^{k+1} r\right)^{N}\right)^{\frac{1}{p^{\prime}}} \\
\leq C r^{\alpha-N+\frac{N}{p^{\prime}}} \\
=C r^{-\frac{N}{q}} .
\end{gathered}
$$

Therefore

$\left|U_{2}(x, r)\right| \leq C r^{-\frac{N}{q}}\|f\|_{L^{p}(K, \lambda)}$ 
From (5) and (6), we have

$$
\left|I_{\alpha} f(x)\right| \leq C\left(r^{\alpha} M f(x)+r^{-\frac{N}{q}}\|f\|_{L^{p}(K, \lambda)}\right)
$$

Minimum of the right-hand side is attained at $r=\left[\frac{\|f\|_{L^{p}(K, \lambda)}}{M f(x)}\right]^{\frac{p}{N}}$. So

$$
\left|I_{\alpha} f(x)\right| \leq C(M f(x))^{\frac{p}{q}}\|f\|_{L^{p}(K, \lambda)}^{1-\frac{p}{q}}
$$

Hence, by the boundedness of Hardy-Littlewood maximal function (3) on $L^{p}(K, \lambda)$ we have

$$
\int_{K}\left|I_{\alpha} f(x)\right|^{q} d \lambda(y) \leq C\|f\|_{L^{p}(K, \lambda)}^{q-p} \int_{K}(M f(y))^{p} d \lambda(y) \leq C\|f\|_{L^{q}(K, \lambda)}^{q} .
$$

The theorem is proved.

Theorem 3.3 Let $(K, *)$ be a commutative hypergroup, with quasi-metric $\rho$ and doubling Haar measure $\lambda$, upper Ahlfors $N$-regular by an identity and let $0<\alpha<N, \frac{1}{q}=1-\frac{\alpha}{N}$ and assume that the maximal operator $M$ is an operator of weak type $(1,1)$ on $(K, *)$. Then the Riesz potential $(4)$ is an operator of weak type $(1, q)$ on $(K, *)$.

Proof Let $f \in L^{1}(K, \lambda)$. It is clear that

$$
\begin{gathered}
\lambda\left\{x \in K:\left|I_{\alpha} f(x)\right|>2 \beta\right\} \\
\leq \lambda\left\{x \in K:\left|U_{1}(x, r)\right|>\beta\right\}+\lambda\left\{x \in K:\left|U_{2}(x, r)\right|>\beta\right\},
\end{gathered}
$$

where

$$
\begin{gathered}
U_{1}(x, r)=\int_{B(e, r)} \rho(e, y)^{\alpha-N} T^{x} f\left(y^{*}\right) d \lambda(y), \\
U_{2}(x, r)=\int_{K \backslash B(e, r)} \rho(e, y)^{\alpha-N} T^{x} f\left(y^{*}\right) d \lambda(y) .
\end{gathered}
$$

Further, from inequality (5) we derive that

$$
\begin{gathered}
\beta \lambda\left\{x \in K:\left|U_{1}(x, r)\right|>\beta\right\}=\beta \int_{\left\{x \in K:\left|U_{1}(x, r)\right|>\beta\right\}} d \lambda(y) \\
\leq \beta \int_{\left\{x \in K: C r^{\alpha} M f(x)>\beta\right\}} d \lambda(y) \\
=\beta \lambda\left\{x \in K: M f(x)>\frac{\beta}{C r^{\alpha}}\right\} \\
\leq \beta \frac{C r^{\alpha}}{\beta} \int_{K}|f(y)| d \lambda(y)=C r^{\alpha}\|f\|_{L_{1}(K, \lambda)}
\end{gathered}
$$

and

$$
\begin{array}{r}
\left|U_{2}(x, r)\right| \leq \int_{K \backslash B(e, r)} \rho(e, r)^{\alpha-N}\left|T^{x} f\left(y^{*}\right)\right| d \lambda(y) \\
\leq r^{\alpha-N} \int_{K \backslash B(e, r)}\left|T^{x} f\left(y^{*}\right)\right| d \lambda(y) \\
\leq C r^{-\frac{N}{q}} \int_{K}|f(y)| d \lambda(y)=C r^{-\frac{N}{q}}\|f\|_{L_{1}(K, \lambda)} .
\end{array}
$$

Thus, if $\beta=r^{-\frac{N}{q}}\|f\|_{L_{1}(K, \lambda)}$, then $\left|U_{2}(x, r)\right| \leq \beta$, and, consequently, $\lambda\left\{x \in K:\left|U_{2}(x, r)\right|>\beta\right\}=0$. Thus

$$
\lambda\left\{x \in K:\left|I_{\alpha} f(x)\right|>2 \beta\right\}
$$




$$
\begin{gathered}
\leq \frac{C}{\beta} r^{\alpha}\|f\|_{L_{1}(K, \lambda)} \\
=C r^{\alpha+\frac{N}{q}}=C r^{N}=C \beta^{-q}\|f\|_{L_{1}(K, \lambda)}^{q} \\
\leq\left(\frac{C}{\beta}\|f\|_{L_{1}(K, \lambda)}\right)^{q} .
\end{gathered}
$$

The theorem is proved.

\section{Acknowledgements}

This work was supported by the Science Development Foundation under the President of the Republic of Azerbaijan Grant EIF-2012-2(6)-39/10/1. The author would like to express his thanks to Academician Akif Gadjiev for valuable remarks.

\section{References}

[1] W. R. Bloom, H. Heyer, Harmonic analysis of probability measures on hypergroups, de Gruyter Stud. Math., Vol. 20, Walter de Gruyter \& Co., Berlin, 1995.

[2] R. R. Coifman and G. Weiss, Analyse harmonique non-commutative sur certains espaces homogènes.(French) Lecture Notes in Math., Vol.242, Springer-Verlag, Berlin-New York, 1971.

[3] A. D. Gadjiev, "On generalized potential-type integral operators", Functiones et Approximatio, UAM, Vol.25 (1997), pp. 37-44.

[4] A. D. Gadjiev, M. G. Hajibayov, "Inequalities for B-convolution operators", TWMS J. Pure Appl. Math., Vol.1, No.1, (2010), pp.41-52.

[5] J. Garcia-Cuerva, A. E. Gatto, "Boundedness properties of fractional integral operators associated to non-doubling measures", Studia Math., Vol.162, (2004), pp.245261 .

[6] G. Gaudry, S. Giulini, A. Hulanicki, A. M. Mantero, "Hardy-Littlewood maximal function on some solvable Lie groups", J. Austral. Math. Soc. Ser. A, Vol.45, No.1, (1988), pp.78-82.

[7] G. Gigante, "Transference for hypergroups", Collect. Math., Vol.52, No.2, (2001), pp.127-155.

[8] V. S.Guliyev, "On maximal function and fractional integral, associated with the Bessel differential operator", Math. Inequal. Appl., Vol.6, No.2, (2003), pp.317-330.

[9] V. S. Guliyev, M.N. Omarova, "On fractional maximal function and fractional integral on the Laguerre hypergroup", J. Math. Anal. Appl., Vol.340, No.2, (2008), pp.1058-1068.

[10] V. S. Guliyev, Y. Y. Mammadov, "On fractional maximal function and fractional integrals associated with the Dunkl operator on the real line", J. Math. Anal. Appl., Vol.353, (2009), pp.449-459.

[11] M.G.Hajibayov, " $\left(L_{p} ; L_{q}\right)$ properties of the potential-type integrals associated to non-doubling measures", Sarajevo J. Math., Vol.2, No.15, (2006), pp.173-180.

[12] M. G. Hajibayov, S. G. Samko, "Weighted estimates of generalized potentials in variable exponent Lebesgue spaces on homogeneous spaces" Operator Theory: Adv. and Appl., Vol.210, (2010), pp.107-122.

[13] M. G. Hajibayov, S. G. Samko, "Generalized potentials in variable exponent Lebesgue spaces on homogeneous spaces", Math. Nachr., Vol.284, No.1, (2011), pp.53-66.

[14] M.G.Hajibayov, "Boundedness of the Dunkl convolution operators", An. Univ. Vest Timis,. Ser. Mat.-Inform., Vol.49, No.1, (2011), pp.49-67.

[15] M.G.Hajibayov, "Inequalities for convolutions of functions on commutative hypergroups", Azerb. J. Math., Vol.4, No.1, (2014), pp.92-107. arXiv preprint arXiv:1307.4948, 2013.

[16] L. Hedberg, "On certain convolution inequalities", Proc. Amer. Math. Soc., Vol.36, (1972), pp.505-510.

[17] R. L. Jewett, "Spaces with an abstract convolution of measures", Adv. in Math., Vol.18, No.1, (1975), pp.1-101. 
[18] V. M. Kokilashvili, A. Kufner, "Fractional inteqrals on spaces of homogeneous type", Comment. Math. Univ. Carolinae, Vol.30, No.3, (1989), pp.511-523.

[19] V. Kokilashvili, A. Meskhi, "Fractional integrals on measure spaces", Frac. Calc. Appl. Anal., Vol.4, No.1, (2001), pp.1-24.

[20] M. Lashkarizadeh Bami, "The semisimplicity of $L^{1}(K, w)$ of a weighted commutative hypergroup K", Acta Math. Sinica, Vol.24, No.4, (2008), pp.607610.

[21] E. Nakai, "On generalized fractional integrals", Taiwanese Math. J., Vol.5, No.3, (2001), pp.587-602.

[22] E. Nakai, H. Sumitomo, "On generalized Riesz potentials and spaces of some smooth functions", Sci. Math. Japonicae, Vol.54, No.3, (2001), pp.463-472.

[23] R. Spector, "Measures invariantes sur les hypergroupes(French)", Trans. Amer. Math. Soc., Vol.239, (1978), pp.147-165.

[24] E. Stein, Singular integrals and diferentiability properties of functions, Princeton Mathematical Series, No. 30 Princeton University Press, Princeton, N.J., 1970. 